\title{
Comparative analysis of management of acute cholecystitis during the SARS-CoV-2 coronavirus pandemic
}

\begin{abstract}
Introduction: COVID-19 infection has spread throughout the world and is considered a pandemic. Since its appearance, the number of non-COVID-19 patients admitted to hospitals has decreased and patients differ care for emergency diseases. We analyze the impact of the SARS-CoV-2 coronavirus pandemic on the management of acute cholecystitis.

Material and methods: Retrospective observational study that includes all patients diagnosed with acute cholecystitis during the SARS-CoV-2 coronavirus pandemic (period between March 11th and June 21st, 2020) and patients diagnosed with acute cholecystitis in the same period, the previous year in our center. Patient's features, management, postoperative complications and mean hospital stay were compared.

Results: In 2020, 19 patients with acute cholecystitis were diagnosed compared to 21 who were registered in the same period in 2019. The mean number of days from symptoms onset in 2020 was $2.42 \pm 1.8$ days, while in 2019 it was $3.5 \pm 3.1$ days $(\mathrm{p}=0.32)$. The percentage of cholecystectomies, percutaneous cholecystostomies and conservative management was similar in both periods. Among patients who underwent cholecystectomy in 2020, $37.5 \%$ had no complications, $62.5 \%$ had accidental opening of the gallbladder, and none had bleeding. Among patients who underwent cholecystectomy in $2019,81.8 \%$ had no complications, $9.09 \%$ had accidental opening of the gallbladder, and $9.09 \%$ presented bleeding. The mean stay in 2020 was $4.21 \pm 3.2$ days, compared to $8.57 \pm 7.4$ days in 2019 $(\mathrm{p}=0.005)$. Two patients of 19 diagnosed with acute cholecystitis in 2020 had COVID-19 disease.

Conclusion: The mean stay of the patients was shorter in 2020 period. These results can be explained by an early surgical management. So, early laparoscopic cholecystectomy should be considered as a treatment for acute cholecystitis in COVID-19 times if the clinical and hospital situation allows it. We found no differences in the number of patients diagnosed with acute cholecystitis between the two periods, nor in the mean number of days from the onset of symptoms.
\end{abstract}

Keywords: acute cholecystitis, SARS-CoV-2, COVID19, coronavirus, pandemic, management
Volume 9 Issue 2 - 202|

\author{
Belén Matías-García, Ana Sánchez-Gollarte, \\ Ana Quiroga-Valcárcel, Fernando Mendoza- \\ Moreno, Javier Mínguez-García, Cristina Vera- \\ Mansilla, Manuel Díez-Alonso, Inmaculada \\ Lasa-Unzué, Alberto Gutiérrez-Calvo \\ Department of General and Digestive Surgery, Príncipe de \\ Asturias Teaching Hospital, Spain
}

Correspondence: Belén Matías-García, Carretera Alcalá-Meco S/N 28805, Alcalá de Henares, Madrid, Spain, Email belenmg3@gmail.com

Received: January 25, 2021 | Published: March 15, 2021
Abbreviations: ICU, intensive care unit; ESTES, European association for trauma and emergency surgery; SAGES, society of American gastrointestinal and endoscopic surgeons; CCT, chest computed tomography; ED, emergency department; CT, computed tomography

\section{Introduction}

Coronavirus disease 2019 (COVID-19) is an infectious respiratory disease caused by the new severe acute respiratory syndrome coronavirus 2 (SARS-CoV-2). ${ }^{1}$ Since its emergence in December 2019, COVID-19 infection has spread worldwide and is considered a pandemic as of 11 March 2020 according to the World Health Organization. ${ }^{2}$ The rapid spread of the pandemic, the ease of personto-person transmission, the high mortality rate and the fear of transmission in hospital led to a decrease in the number of COVID19-free patients admitted to hospitals. ${ }^{1}$ This situation suggested that patients may be deferring care for serious diseases whose prevalence would be expected to be stable, as in the case of acute cholecystitis. ${ }^{3}$ Acute cholecystitis is one of the most frequent pathologies in daily clinical surgical practice. Early cholecystectomy within 7 days of symptom onset is the treatment of choice for acute cholecystitis. ${ }^{4}$ In a
SARS-CoV-2 pandemic, this delay in care suggests increased disease severity and complication rates. ${ }^{5}$ In addition, the rapid spread of the disease led to a dramatic increase in the number of patients admitted to emergency departments and patients admitted to hospitals. ${ }^{1}$ The need to increase hospital bed capacity and intensive care unit (ICU) capacity for these patients necessitated a reorganization of the various hospital departments. Surgical staff were mobilised to care for patients with COVID-19 disease. ${ }^{6}$ During this period, the European Association for Trauma and Emergency Surgery (ESTES) recommended postponing elective surgery cases to a later date, except in some identified cases. ${ }^{1,6}$ The need for surgery in some identified cases and the possibility of performing surgery was discussed in a multidisciplinary team.

However, in emergency surgery the recommendations to be followed were less clear and are still controversial today. The British Intercollegiate General Surgical Guideline on COVID-19 stated that during the COVID-19 pandemic, non-surgical treatment should be applied whenever possible (as in the case of early appendicitis and acute cholecystitis). ${ }^{7,8}$ Other surgical societies, such as the Society of American Gastrointestinal and Endoscopic Surgeons (SAGES), have recommended a more patient- and hospital-centered approach. ${ }^{7,9}$ In this study, we compared acute cholecystitis diagnosed during the 
COVID-19 pandemic period with the same period of the previous year. We aim to analyze whether there are differences in the epidemiology, clinical or management of acute cholecystitis resulting from the effect of the COVID-19 outbreak on this emergency pathology.

\section{Material and methods}

Retrospective observational study including all patients diagnosed with acute cholecystitis during the SARS-CoV-2 coronavirus pandemic (period from 11 March to 21 June 2020) and patients diagnosed with acute cholecystitis in the same period the previous year in our centre (population catchment area, 243,000 inhabitants). All patients diagnosed with acute cholecystitis who were admitted to our hospital during the SARS-CoV-2 coronavirus pandemic period were screened for COVID19 disease by polymerase chain reaction (PCR) and for antibodies to SARS-CoV-2 coronavirus by serology. Inclusion criteria for the study were all patients older than 18 years with a diagnosis of acute cholecystitis on imaging (ultrasound/ computed tomography) Figure 1. Exclusion criteria were patients younger than 18 years, pregnant women, associated pancreatitis or ICU admission.

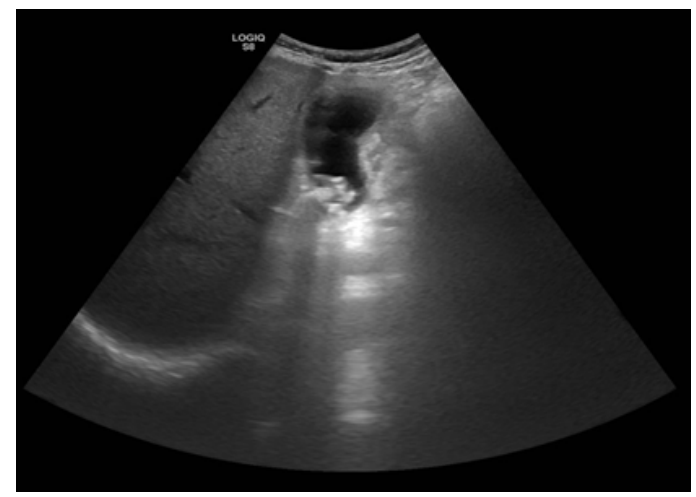

Figure I Distended gallbladder with thickening of the gallbladder wall and lithiasis inside.

Data were analyzed with IBM SPSS Statistic ${ }^{\circledR}$ version 25 . For categorical variables, the proportion of each category with respect to the total number of patients was calculated. For quantitative variables, the mean and standard deviation were studied. The Mann-Whitney U test was used to compare means.

The variables analyzed were gender, age, days since symptom onset, treatment (cholecystectomy, percutaneous cholecystostomy or non-operative management), morbidity and mortality, and mean hospital stay. Non-operative management included treatment with mainly absolute diet and empirical antibiotherapy. Data were tabulated and analyzed with IBM SPSS Statistic ${ }^{\circledR}$ version 25.

\section{Results}

In the established period of 2020, 19 patients were diagnosed with acute cholecystitis compared to 21 in the same period of 2019 . The mean age was $69 \pm 16.1$ years in 2020 and $75.2 \pm 11.8$ years in 2019 . The diagnosis of acute cholecystitis was more prevalent in males in both time periods (57.9\% in 2020 and $61.9 \%$ in 2019). The mean number of days since symptom onset in 2020 was $2.42 \pm 1.8$ days, while in 2019 it was $3.5 \pm 3.1$ days. No statistically significant differences were found between the two time periods (Mann-Whitney U-test 154.5, $\mathrm{p}=0.32)$. Cholecystectomy was performed in $52.6 \%(\mathrm{n}=10)$ of patients diagnosed in 2020, non-surgical management was performed in $42.1 \%$ $(\mathrm{n}=8)$ of patients and percutaneous cholecystostomy was performed in $5.3 \%(\mathrm{n}=1)$ of patients; cholecystectomy was performed in $52.4 \%$ $(n=11)$ of patients diagnosed in 2019 , non-surgical management was performed in $38.1 \%(\mathrm{n}=8)$ of patients and cholecystostomy was performed in $9.5 \%(n=2)$ of patients.

Among patients who underwent cholecystectomy in 2020,37.5\% $(n=3)$ had no complications, $62.5 \%(n=7)$ had an accidental opening of the gallbladder and none had a hemorrhage. Among patients undergoing cholecystectomy in $2019,81.8 \%(\mathrm{n}=9)$ had no complications, $9.09 \%$ $(\mathrm{n}=1)$ had accidental opening of the gallbladder and $9.09 \%(\mathrm{n}=1)$ had bleeding. Bile duct injuries were not recorded in any of the periods. The mean length of stay in 2020 was $4.21 \pm 3.2$ days, compared to $8.57 \pm 7.4$ days in 2019. Statistically significant differences were found between the two periods (Mann-Whitney U-test 97.5, p=0.005). All postoperative complications recorded in both periods were grade I according to the Clavien Dindo classification. Patients were treated with prophylactic heparin at discharge according to the Caprini score. No readmissions were recorded in either period. No deaths were recorded within 30 days. Only 2 patients of the 19 diagnosed with acute cholecystitis in 2020 had COVID-19 disease. None of these patients had morbidity associated with the initial COVID-19 disease process.

\section{Discussion}

Adecrease in the number of emergency department(ED) admissions for non-COVID-19 disease has been reported in some studies. ${ }^{1,2,5} \mathrm{~A}$ delay in ED admissions compared to non-pandemic times has also been reported. ${ }^{1,2,5}$ Boyle et al., ${ }^{5}$ reported a reduction in acute cholecystitis volume of $39.2 \%$ comparing the pre-closure period and the closure period. ${ }^{5}$ A delay in the presentation of patients with cholecystitis undergoing acute laparoscopic cholecystectomy is more likely to lead to conversion to an open procedure, resulting in increased morbidity and longer length of stay. ${ }^{5}$ In our study, we found no difference in the number of ED admissions for acute cholecystitis (19 patients in 2020 versus 21 patients in 2019). We also found no statistically significant differences in the mean days from symptom onset to ED admission of our patients [ 2.42 days in 2020 vs. 3.5 days in $2019(\mathrm{p}=0.32)$ ]. Following ESTES recommendations, ${ }^{6}$ the management of ED general surgery patients was reviewed by two surgeons and care was taken not to delay interventions and to maintain their quality. The percentage of patients undergoing emergency cholecystectomy was $52.6 \%$ in the group of patients with acute cholecystitis during the pandemic and $52.4 \%$ in the control group during the non-coviral period. These results differ from those obtained in other studies such as Surek et al., ${ }^{1}$ in which acute cholecystitis was one of the pathologies in which a greater decrease in emergency surgery was observed. Laparoscopic cholecystectomy is the treatment of choice for acute cholecystitis in non-pandemic times Figure 2.

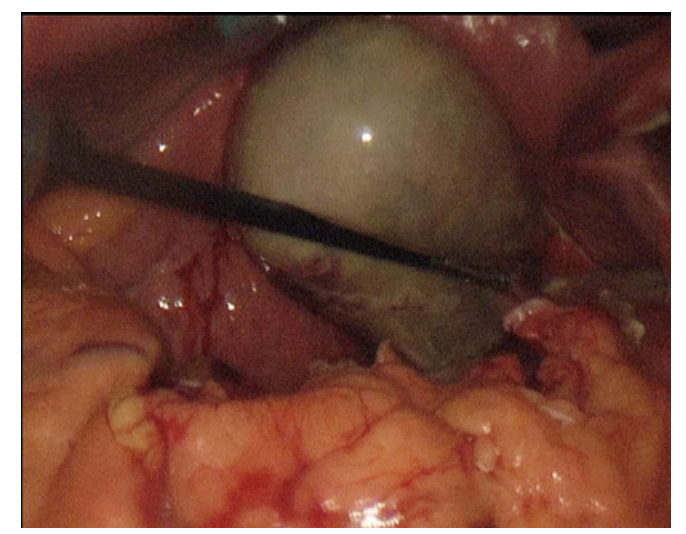

Figure 2 Gallbladder with signs of acute cholecystitis during the performance of a laparoscopic cholecystectomy. 
However, the recommendation of early surgery could be questioned due to limitations related to COVID-19. ${ }^{10}$ In addition, the most appropriate surgical approach is subject to debate in pandemic times. Initial advice seemed to advise against the use of laparoscopy. ${ }^{11}$ In previous research there appears to be a greater potential for virus spread with laparoscopic surgery due to aerosolisation by the pneumoperitoneum and the steam formed by heat-generating cauterization devices. ${ }^{11,12}$ However, according to the Society of American Gastrointestinal and Endoscopic Surgeons (SAGES), there is currently no evidence to suggest that this effect occurs with the SARS-CoV-2 coronavirus. ${ }^{11,12}$ In addition, SAGES also noted that the benefits of laparoscopic surgery, such as reduced hospital stay, should also be taken into account. ${ }^{12}$ Another aspect to be highlighted in both approaches is the importance of the correct use of protective equipment and the impact this can have on the performance of the surgical procedure. ${ }^{13,14}$ Some authors suggest a more significant impact on the surgeon during open surgery due to reduced comfort, communication difficulties and reduced visibility. ${ }^{15}$

Therefore, non-surgical treatment should be considered as a management alternative. ${ }^{10}$ The Tokyo 2018 guidelines recommend non-surgical treatment only in patients with significant clinical deterioration, such as patients with ASA 3 or those with a Charlson scale $>5$ (or $>3$ in those with Tokyo grade III acute cholecystitis). ${ }^{16,17}$ Although the percentages of non-surgical treatment were similar in our study (42.1\% of patients in 2020 and $38.1 \%$ of patients in 2019), in a pandemic situation it is worth reviewing non-surgical treatment. ${ }^{16}$ Percutaneous cholecystostomy may be another alternative in critically ill patients. ${ }^{10}$ However, the Tokyo 2018 guidelines recommend it only in patients with acute grade III cholecystitis and predictive factors for poor prognosis and/or cardiovascular or renal organ failure that is not rapidly reversible after admission. ${ }^{17}$ In our study, percutaneous cholecystostomy was performed in a similar percentage of patients in both time periods $(5.3 \%$ of patients in 2020 and in $9.5 \%$ of patients in 2019). The indication for percutaneous cholecystostomy in these 3 patients was acute grade III cholecystitis with associated renal failure not rapidly reversible with medical treatment. Interventional radiology does not provide 24-hour service in our hospital, which may have influenced the management of these patients.

In patients undergoing emergency cholecystectomy, we found notable differences in surgical complications between the two periods. During the pandemic period, we recorded $62.5 \%$ of accidental opening of the gallbladder during the procedure. During the non-pandemic period, we reported only $9.09 \%$ of accidental opening of the gallbladder. These differences can be explained by the prospective data collection during the 2020 period versus the retrospective data collection in the 2019 period. We found only one case of hemorrhage during 2019 No bile duct injuries or other surgical complications were recorded in either period. Length of stay was shorter during the pandemic period with a statistically significant difference [4.21 \pm 3.2 days in 2020 versus 8.57 days in $2019(\mathrm{p}=0.005)$ ]. These results are similar to those obtained in other studies. ${ }^{2}$ Laparoscopic cholecystectomy reduces the duration of hospital admission. ${ }^{4}$ Therefore, these results can be explained by early surgical management of laparoscopic cholecystectomy. In contrast to our study, Boyle et al., ${ }^{5}$ reported a longer length of stay in patients diagnosed with acute cholecystitis during the closure period. ${ }^{5}$ However, the management of their patients was mainly non-operative management, ${ }^{5}$ which differs from our mainly surgical management. An early hospital discharge in times of SARS-CoV-2 coronavirus pandemic reduces the risk of hospitalacquired infection. Thus, early laparoscopic cholecystectomy should be considered as treatment of acute cholecystitis if the clinical and hospital situation allows it.
Only two patients with acute cholecystitis were concomitantly diagnosed with COVID-19 disease. Both patients were asymptomatic for COVID-19 disease during their hospital stay. Diagnosis of COVID-19 disease was made by PCR screening and chest radiography in all patients admitted to the hospital. No patients were screened by chest computed tomography (CCT). However, some authors recommend CCT scanning for screening of COVID-19 disease if the patient needs another diagnostic computed tomography (CT) scan.18 One of these patients was diagnosed with acute alithiasic cholecystitis by CT. The absence of gallbladder stones invites us to consider whether acute cholecystitis was a complication of COVID-19 disease or whether they were two independent concomitant diseases. Bruni et al., ${ }^{19}$ described a case of acute gangrenous cholecystitis as a late complication in a patient with COVID-19 disease. ${ }^{19}$ The cause of acute cholecystitis could be thrombosis of the gallbladder vessels, described in the pathological anatomy, derived from a systemic inflammatory response to COVID-19 disease. ${ }^{19}$ However, the precise mechanism of acute cholecystitis in patients with COVID-19 is unknown. ${ }^{20}$ Therefore, the presentation of acute cholecystitis as a complication of COVID-19 disease awaits the development of new studies that may prove or disprove this hypothesis. ${ }^{20}$

\section{Conclusion}

The present study analyzes the evolution of patients diagnosed with acute cholecystitis during the SARS-CoV-2 pandemic. The mean length of stay of patients was shorter in the 2020 period. These results may be explained by earlier surgical management of laparoscopic cholecystectomy. A shorter hospital stay reduces the risk of nosocomial infection. Thus, early laparoscopic cholecystectomy should be considered as a treatment for acute cholecystitis in COVID-19 times if the clinical and hospital situation allows it. Contrary to what might be expected, we found no differences in the number of patients diagnosed with acute cholecystitis between the two periods, nor in the mean number of days from symptom onset. Treatment of patients was similar in the COVID-19 pandemic period to the same period last year. However, non-surgical treatment of acute cholecystitis and percutaneous cholecystectomy should be considered as an alternative to early laparoscopic cholecystectomy in times of pandemic. Finally, the presentation of acute cholecystitis as a complication of COVID-19 disease remains to be proven and further studies are needed to prove or disprove this hypothesis.

\section{Funding}

None.

\section{Acknowledgments}

None.

\section{Conflicts of interest}

The authors declare that there are no conflicts of interest.

\section{References}

1. Surek A, Ferahman S, Gemici E, et al. Effects of COVID-19 pandemic on general surgical emergencies: are some emergencies really urgent? Level 1 trauma center experience. Eur J Trauma Emerg Surg. 2020;1-6.

2. Cano-Valderrama O, Morales X, Ferrigni CJ, et al. Acute care surgery during the COVID-19 pandemic in Spain: changes in volume, causes and complications. A multicentre retrospective cohort study. Int J Surg. 2020;80:157-161.

3. Walker LE, Heaton HA, Monroe RJ, et al. Impact of the SARS-CoV-2 pandemic on emergency department presentations in an integrated health system. Mayo Clin Proc. 2020;95(11):2395-2407. 
4. Halpin V. Acute cholecystitis. BMJ Clin Evid. 2014:0411.

5. Boyle LI, Boyle A, Jay S, et al. COVID-19 lockdown impact on common general surgical acute presentations to a regional centre in New Zealand. N Z Med J. 2020;133(1525):96-105.

6. Coimbra R, Edwards S, Kurihara H, et al. European society of trauma and emergency surgery (ESTES) recommendations for trauma and emergency surgery preparation during times of COVID-19 infection. Eur J Trauma Emerg Surg. 2020;46(3):505-510.

7. Campanile FC, Podda M, Arezzo A, et al. Acute cholecystitis during COVID-19 pandemic: a multisocietary position statement. World J Emerg Surg. 2020;15(1):38

8. https://www.rcseng.ac.uk/coronavirus/joint-guidance-for-surgeons-v2

9. https://www.sages.org/category/covid-19

10. De Simone B, Chouillard E, Di Saverio S, et al. Emergency surgery during the COVID-19 pandemic: what you need to know for practice. Ann R Coll Surg Engl. 2020;102(5):323-332.

11. Di Saverio S, Khan M, Pata F, et al. Convert to open: the new paradigm for surgery during COVID-19? Br J Surg. 2020;107(7):e194.

12. Di Saverio S, Khan M, Pata F, et al. Laparoscopy at all costs? No now during COVID-19 outbreak and not for acute care surgery and emergency colorectal surgery: a practical algorithm from a hub tertiary teaching hospital in Northern Lombardy, Italy. J Trauma Acute Care Surg. 2020;88(6):715-718.
13. Yánez Benítez C, Ribeiro MAF Jr, Alexandrino H, et al. International cooperation group of emergency surgery during the COVID-19 pandemic. Eur J Trauma Emerg Surg. 2020:1-9.

14. BenÍtez CY, Pedival AN, Talal I, et al. Adapting to an unprecedented scenario: surgery during the COVID-19 outbreak. Rev Col Bras Cir. 2020;47:e20202701.

15. Yánez Benítez C, Güemes A, Aranda J, et al. Impact of personal protective equipment on surgical performance during the COVID-19 pandemic. World J Surg. 2020;44(9):2842-2847.

16. Parreira JG, Campos T, Antunes PSL, et al. Management of non traumatic surgical emergencies during the COVID-19 pandemia. Rev Col Bras Cir. 2020;47:e20202614

17. Okamoto K, Suzuki K, Takada T, et al. Tokyo guidelines 2018: flowchart for the management of acute cholecystitis. J Hepatobiliary Pancreat Sci. 2018;25(1):55-72.

18. Lima DS, Ribeiro MAF Jr, Gallo G, et al. Role of chest CT in patients with acute abdomen during the COVID-19 era. Br J Surg. 2020;107(7):e196.

19. Bruni A, Garofalo E, Zuccalà V, et al. Histopathological findings in a COVID-19 patient affected by ischemic gangrenous cholecystitis. World J Emerg Surg. 2020;15(1):43.

20. Ying M, Lu B, Pan J, et al. COVID-19 with acute cholecystitis: a case report. BMC Infect Dis. 2020;20(1):437. 